\title{
The Presence of Renal IgG Deposits in Necrotizing Crescentic Glomerulonephritis Associated with ANCA Is Not Related to Worse Renal Clinical Outcomes
}

\author{
Caroline Dudreuilh $^{\mathrm{a}}$ Fadi Fakhouri $^{\mathrm{b}}$ Cécile Vigneau ${ }^{\mathrm{c}}$ Jean-François Augusto ${ }^{\mathrm{d}}$ \\ Marie-Christine Machet ${ }^{\mathrm{e}}$ Nolwenn Rabot $^{\mathrm{a}}$ Marion Chapal $^{\mathrm{b}}$ Vianney Charpy ${ }^{\mathrm{b}}$ \\ Christelle Barbet $^{\mathrm{a}}$ Matthias Büchler ${ }^{\mathrm{a}} \quad$ Jean-Michel Halimi ${ }^{\mathrm{a}} \quad$ Philippe Gatault $^{\mathrm{a}}$ \\ a Department of Nephrology - Hypertension, Dialysis, Transplantation, CHRU, Tours, France; ${ }^{b}$ Department of \\ Nephrology and Immunology and INSERM UMR S-1064, CHU, Nantes, France; 'Department of Nephrology, \\ CHU Hôpital de Pontchaillou, Rennes, France; 'Department of Nephrology-Dialysis-Transplantation, $\mathrm{CHU}$,

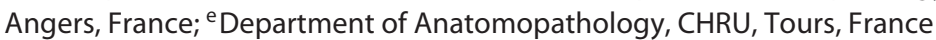

\section{Keywords}

Antineutrophil cytoplasmic antibodies - Glomerulonephritis . Immunoglobulin G · Kidney · Systemic vasculitis

\begin{abstract}
Introduction: Classical pauci-immune necrotizing crescentic glomerulonephritis (CGN) associated with antineutrophil cytoplasmic autoantibodies (ANCA) is characterized by the absence of renal immunoglobulin (Ig) deposits. However, IgG deposits can sometimes be present. We wanted to assess whether necrotizing CGN with IgG deposits is associated with a more severe presentation and outcome than necrotizing CGN without IgG deposits. Methods: Between November 2008 and August 2013, we retrospectively identified 158 patients from four centers who had necrotizing CGN due to primary ANCA-associated systemic vasculitis. Glomerular IgG deposits were found in 18 (11\%) patients (group 1). For each patient in group 1 , we selected 2 patients with classical pauci-immune necrotizing CGN with the nearest date of diagnosis in the same center (group 2, $n=36$ ). We assessed clinical, biological, and pathological characteristics in both
\end{abstract}

\begin{tabular}{ll}
\hline KARGER & (c) 2019 The Author(s) \\
& Published by S. Karger AG, Basel \\
karger@karger.com & This article is licensed under the Creative Commons Attribution- \\
www.karger.com/kdd & NonCommercial-NoDerivatives 4.0 International License (CC BY- \\
NC-ND) (http://www.karger.com/Services/OpenAccessLicense). \\
Usage and distribution for commercial purposes as well as any dis- \\
tribution of modified material requires written permission.
\end{tabular}

groups. Results: Baseline characteristics were similar in both groups, and most patients had ANCA-associated vasculitis with antibodies to myeloperoxidase (74\%). Deposits displayed moderate to strong staining in 9 patients. As compared with group 2, group 1 exhibited a higher frequency of interstitial fibrosis/tubular atrophy lesions $(p=0.024)$ and lower frequency of acute tubular necrosis $(p=0.046)$. Nevertheless, after a mean follow-up of 30 and 26 months for group 1 and group 2, respectively, Ig G deposits did not affect the renal prognosis or probability of relapse. Finally, the groups did not differ in renal or patient survival. Conclusions: IgG deposits, detected in $11 \%$ of patients with ANCAassociated necrotizing CGN, did not affect renal or patient outcomes.

(c) 2019 The Author(s)

Published by S. Karger AG, Basel

\section{Introduction}

Antineutrophil cytoplasmic antibody (ANCA)-associated vasculitis (AAV), including granulomatosis with polyangiitis, microscopic polyangiitis, and renal limited

Caroline Dudreuilh, MD

Department of Nephrology, Immunology and Transplantation, CHU Tours 2 Boulevard Tonnellé

FR-37000 Tours (France)

E-Mail caroline.dudreuilh@ $@$ kcl.ac.uk 


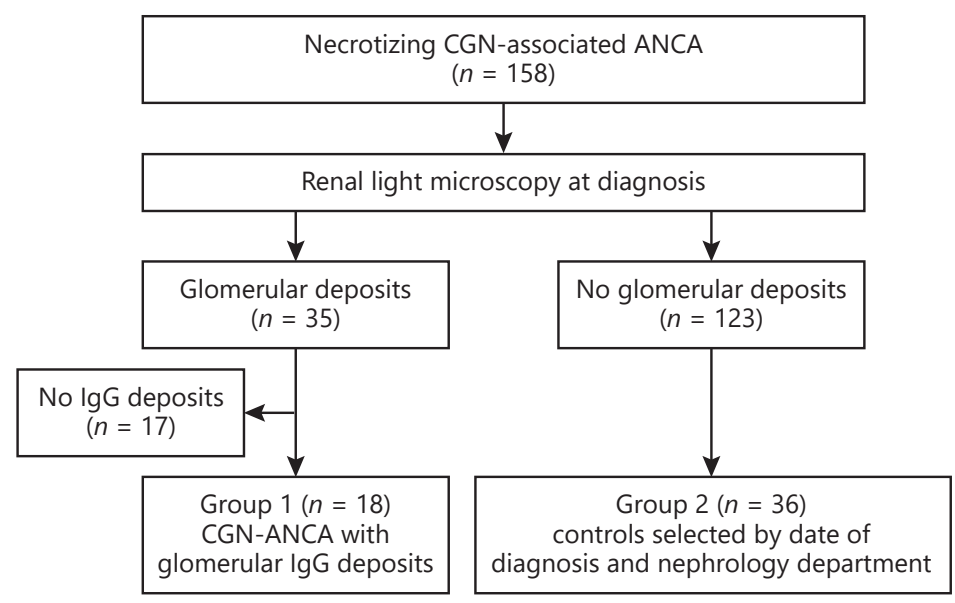

Fig. 1. Flowchart of the study population.

$\mathrm{AAV}$, are multisystemic severe autoimmune diseases [1]. Histological proof of vasculitis remains a pivotal element to establish the diagnosis of AAV [2-4]. The combination of necrotizing crescentic glomerulonephritis (CGN) (most often responsible for rapidly progressive GN and observed in about $20-40 \%$ of patients with AAV) with antimyeloperoxidase (MPO) and antiproteinase 3 (PR3) autoantibodies is deemed sufficient for a definitive diagnosis of ANCA-associated renal vasculitis [5-7].

Among CGN cases, ANCA-associated CGN is usually characterized by the absence of immunoglobulin (Ig) deposits in the kidney. Hence, these cases are described as "pauci-immune" in contrast to both immune complexmediated glomerulonephritis (GN) characterized by Ig and complement deposits or GN with linear deposits of anti-glomerular basement membrane antibodies. However, a few studies have reported some glomerular immune deposits seen on light microscopy (immunofluorescence) or electron microscopy in ANCA-associated CGN [8-10]. Importantly, these studies have suggested that immune deposits may be associated with more severe renal damage, namely, a possible higher proportion of crescents, poorer renal function, and greater proteinuria at the time of diagnosis [4, 8-11]. These studies suggested that the presence of glomerular immune deposits in patients with ANCA-associated CGN could be associated with worse renal outcome.

Nevertheless, the impact of immune deposits in ANCA-associated CGN remains disputed because the long- term renal outcome was not reported [8], not compared to other patients with pauci-immune ANCA-associated GN [9], or affected only a few patients [10]. In those studies, chronic lesions, such as sclerotic crescents or tubular atrophy and interstitial fibrosis, which have been described as negative prognostic factors $[12,13]$, were not more frequent in patients with deposits than without immune deposits $[9,10]$. Moreover, the impact of immune deposits was always analyzed independently of their isotype, as IgM, IgG, and IgA deposits can also be observed. IgG deposits are the most frequent $[8,9]$, but they are also often found in patients without deposits seen on electronic microscopy [8]. In contrast, IgA and/or IgM deposits are usually not found in the absence of immune deposits seen on electronic microscopy and can involve additional and different pathogenic pathways [8], such as the presence of ANCA-associated IgA [13]. In this study, we evaluated whether the presentation and outcomes of patients with ANCA-associated CGN were affected by the presence of glomerular IgG deposits.

\section{Materials and Methods}

Selection of Patients

In this retrospective study, among 158 patients with ANCAassociated CGN diagnosed between November 2008 and August 2013 in four French nephrology departments (Tours, Nantes, Rennes, and Angers), 35 patients showed glomerular immune deposits on immunofluorescence microscopy (Fig. 1). Overall, 18 of 
158 patients (11\%) had IgG deposits (group 1), whereas the remaining 17 presented other Ig isotypes deposits without IgG deposits and were excluded. Although IgG deposits can be observed in diabetic nephropathy, none of the patients in group 1 were diabetic [8]. Moreover, all patients in this study were tested negative for both anti-double-stranded DNA antibody and anti-glomerular basement membrane antibody, usually found in lupus nephritis and anti-glomerular basement membrane disease, respectively.

Next, for each patient from group 1, we selected 2 control patients, as per standard practice, with pauci-immune ANCA-associated CGN without IgG deposits. Thus, we included 36 of the remaining 123 patients (group 2) with renal biopsy performed just before and just after every patient in group 1 within the same nephrology department to reduce the bias associated with change in treatment practice.

\section{Clinical, Biological, and Treatment Data}

The patient records were individually reviewed to determine the type of AAV according to the revised Chapel Hill classification [1] and to determine the Birmingham Vasculitis Activity Score (BVAS) at diagnosis [14]. Multiorgan involvement was defined as the involvement of three or more organs.

The following demographic and clinical data were recorded: age, sex, arterial blood pressure, smoking status, professional activity, existence of a triggering factor, fever, fatigue, weight loss, arthralgia, and myalgia. To evaluate the initial severity of CGN, we recorded proteinuria, hematuria, serum creatinine, and estimated glomerular filtration rate (eGFR) values using the Modification of Diet in Renal Disease (MDRD) equation and the requirement for dialysis treatment [15]. We also recorded immunological variables: titers of anti-MPO and anti-PR3 antibodies, C3 and C4 complement levels, and the presence of anti-dsDNA antibodies, rheumatoid factor, or anti-glomerular basement antibody.

Initial, rescue, and maintenance drug therapy were recorded: corticosteroids (dose, route of administration, duration), cyclophosphamide (dose, number of infusions, duration), rituximab (dose, number of infusions, duration), azathioprine (dose and duration), and mycophenolate mofetil (MMF) (dose and duration). The number and duration of plasma exchanges were recorded.

The following biological data were collected at diagnosis and at 1 and 5 years: relapse, eGFR, and proteinuria. The end of follow-up was defined as death or date of last visit. Renal survival was assessed by creatinine level or end-stage renal disease at last followup (ESRD: need for chronic dialysis or renal transplantation) and did not include patients who had temporary dialysis but recovered. Relapses were defined as changes in the BVAS score and or apparition of new symptoms/organs involvement. Renal relapse was defined as positivity of a urine dipstick for blood or protein if negative before and/or increase of creatinine which led to a new kidney biopsy with active lesions.

\section{Renal Biopsy}

We retrospectively reviewed the histopathology reports and recorded the following items: total number of glomeruli, number of glomeruli with crescents typed cellular or fibrocellular, number of sclerotic glomeruli, and pathological changes in tubules, interstitium, and blood vessels. Mesangial hypercellularity was noted because it is a prognostic factor of kidney function in AAV [12]. We also retrospectively classified histopathological findings according to the last publication of the European Vasculitis Study Group into four types [16]: focal, sclerotic, mixed, and crescentic. The proportions of crescents and sclerotic glomeruli were calculated as a percentage of the total number of glomeruli present in each biopsy. As in previous reports $[10,13,16]$, interstitial fibrosis and tubular atrophy were semiquantitatively scored from 0 to 3, for absent, mild (1-25\%), moderate (25-50\%), or severe (>50\%), respectively. Acute tubular necrosis was recorded. Chronic vascular lesions unrelated to vasculitis represented either arteriolar hyalinosis or fibrous endarteritis lesions. Finally, we focused on the staining pattern of Ig deposits (linear or granular, focal or diffuse) identified by direct immunofluorescence. To compare our results with previous reports of ANCA-associated GN with IgG deposits [8, 10, 17], we quantified IgG deposit staining from 0 to 4 , as negative, weak, moderate, strong, and very strong, respectively. Additionally, we noted whether other types of deposits were present, such as $\operatorname{IgA}$ and IgM or complement components (C3, C4, C1q). The pathology slides were assessed locally and then the pathology reports were reviewed by the same 2 individuals all way through to insure both consistency and reduce bias.

\section{Statistical Analysis}

Results are expressed as percentage or mean \pm SD for normally distributed variables and median (range) for non-normally distributed variables. Categorical data were compared by $\chi^{2}$ or Fisher's exact test according to sample size. Quantitative data were compared by Mann-Whitney test. Kaplan-Meier curves were used to estimate the probability of patient survival, renal survival (ESRD), and relapse in both groups and were compared by logrank test. Statistical analyses involved using XLSTAT software. A $p<0.05$ was considered statistically significant.

\section{Results}

\section{Patient Characteristics}

Patient characteristics are presented in Table 1. Except for age, demographic characteristics were similar between the groups. The groups did not differ according to delay between presentation and diagnosis, disease activity, or type of AAV. Thus, microscopic polyangiitis was diagnosed in most patients in both groups. Renal limited vasculitis was seen frequently; however, 5 patients in group 1 presented with Raynaud's syndrome and 3 patients with well-characterized autoimmune diseases: rheumatoid arthritis, scleroderma, or ankylosing spondylitis. The groups did not differ according to the incidence of rheumatoid factor or anti-nuclear antibodies. C3 and $\mathrm{C} 4$ complement levels were normal in all patients. MPO-ANCAs were frequent in both groups, especially group $1(16 / 18)$, and PR3-ANCAs were identified in (13/36) $36 \%$ of patients in group 2 . The MPO-ANCA level was more frequently $>100 \mathrm{IU} / \mathrm{L}$ in group 1 as compared to group 2 (75 vs. $41 \%, p=0.009$ ). 
Table 1. Clinical, biological, and pathological characteristics data in all patients ${ }^{1}$

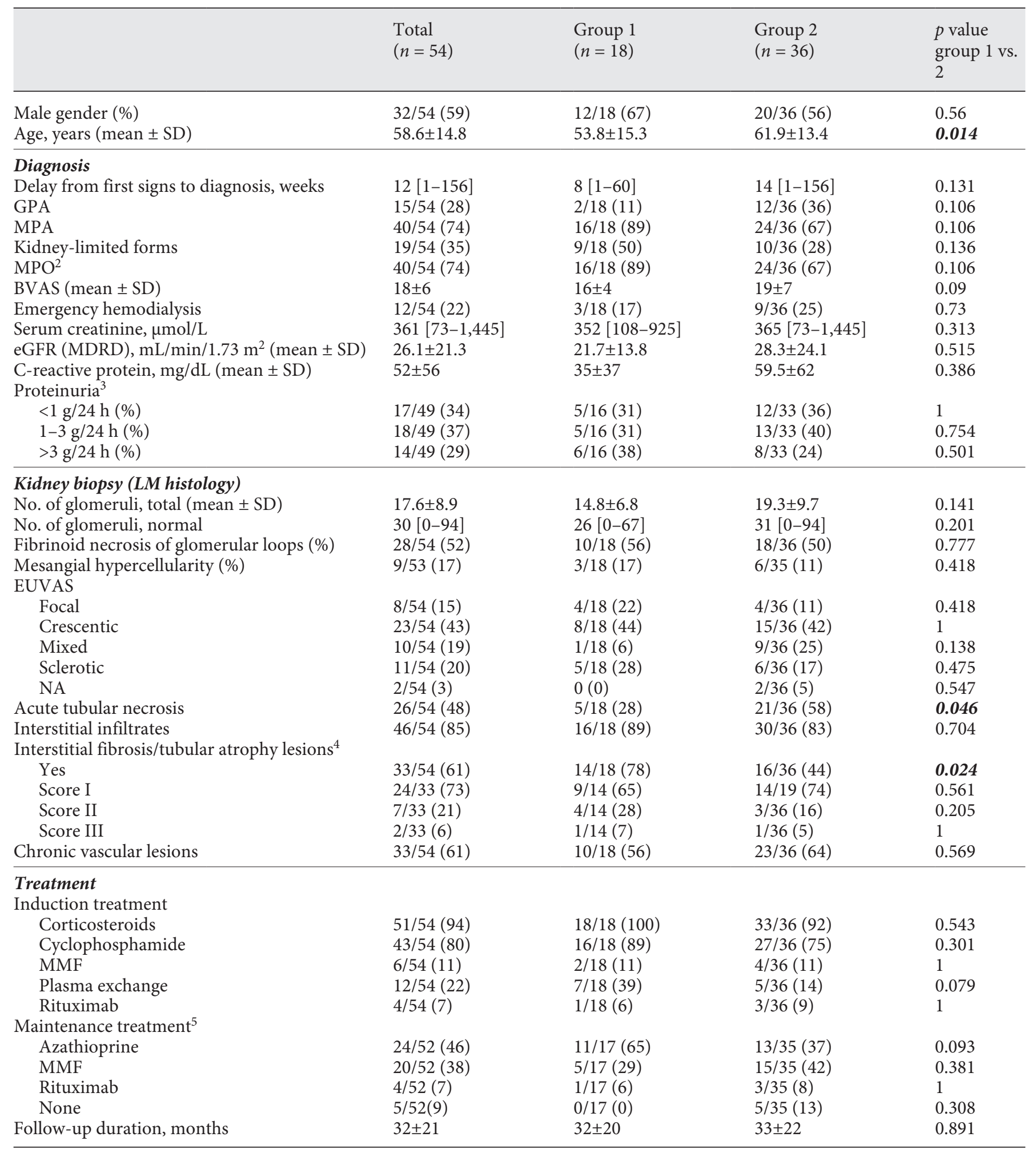


Table 1 (Footnote)

MPA, microscopic polyangiitis; GPA, granulomatosis with polyangiitis; PR3, PR3 antibodies; MPO, anti-myeloperoxidase antibodies; BVAS, Birmingham Vascular Activity Score; eGFR, estimated glomerular filtration rate; MDRD, Modification of Diet in Renal Disease; LM, light microscopy; No. of glomeruli, total number of glomeruli on kidney biopsy; EUVAS scores: score I, mild (1-25\%); score II, moderate (25-50\%); score III, severe ( $>50 \%)$; MMF, mycophenolate mofetil. ${ }^{1}$ Patients with primary ANCA-associated systemic vasculitis with (group 1) and without (group 2) renal immunoglobulin G deposits. ${ }^{2}$ MPO concentration was available in 34 of 36 patients in group $2 .{ }^{3}$ Proteinuria in $\mathrm{g} / 24 \mathrm{~h}$ was available in 16 of 18 (89\%) in group 1 and 33 of 36 (92\%) in group $2 .{ }^{4}$ Tubular atrophy and interstitial fibrosis were not available in 1 patient in group $2 .{ }^{5}$ One patient died before the end of the induction treatment.

Table 2. Clinical and biological characteristics of patients with IgG deposits (group 1)

\begin{tabular}{|c|c|c|c|c|c|c|c|c|c|c|c|}
\hline 1 & $\mathrm{M}$ & 48 & 60 & yes & no & 12 & 23 & 253 & 16.1 & 16.6 & MPO \\
\hline 2 & $\mathrm{M}$ & 48 & 14 & no & no & 15 & 53 & 133 & 0.6 & ND & MPO \\
\hline 5 & $\mathrm{M}$ & 53 & 52 & no & no & 20 & 16 & 370 & 5.34 & 7 & MPO \\
\hline 6 & $\mathrm{~F}$ & 48 & 2 & yes & yes & 22 & 7 & 626 & 1.17 & 0.88 & MPO \\
\hline 7 & $\mathrm{M}$ & 60 & 8 & yes & no & 19 & 14 & 412 & 4.61 & 7.8 & MPO \\
\hline 8 & $\mathrm{~F}$ & 32 & 12 & no & yes & 16 & 12 & 388 & 0.84 & 2.02 & MPO \\
\hline 9 & $\mathrm{M}$ & 74 & NA & yes & no & 12 & 19 & 297 & 1.09 & 1.72 & MPO \\
\hline 14 & $\mathrm{~F}$ & 80 & 1 & yes & no & 12 & 18 & 242 & 3.9 & 4.28 & MPO \\
\hline 15 & $\mathrm{~F}$ & 20 & 14 & no & yes & 21 & 18 & 294 & 2.14 & 1.28 & MPO \\
\hline 16 & $\mathrm{M}$ & 51 & 4 & no & yes & 21 & 24 & 263 & 1.27 & 1.27 & PR3 \\
\hline 17 & $\mathrm{M}$ & 62 & 3 & no & no & 19 & 7 & 715 & 0.91 & 0.25 & PR3 \\
\hline 18 & $\mathrm{~F}$ & 30 & 24 & no & yes & 17 & 55 & 108 & 0.25 & 0.78 & MPO \\
\hline
\end{tabular}

BVAS, Birmingham Vascular Activity Score; eGFR, glomerular filtration rate; MDRD, Modification of Diet in Renal Disease; Pu, proteinuria; MPO, myeloperoxidase; PR3, proteinase 3; NA, not available; ND, not determined.

Except for 1 patient with acute Pseudomonas aeruginosa sepsis and 3 patients with renal limited vasculitis and irreversible kidney damages, all patients received at least one intravenous pulse of methylprednisolone, associated with intravenous pulses of cyclophosphamide $(n=43)$ in the majority of them. In total, 48 of the 52 patients alive after induction therapy received maintenance immunosuppressive drugs. Maintenance treatment was azathioprine, MMF, or rituximab, which were given to 24,20 , and 4 patients, respectively. Steroids with a gradual tapering were additionally given for all but 5 patients.
The groups did not differ according to induction and maintenance regimens. Plasma exchange tended to be performed more frequently in group $1(p=0.079)$.

Clinical and biological data for group 1 patients are presented in Table 2. Hematuria was present at diagnosis in all patients. The two groups were similar with regard to need for dialysis, eGFR, and proteinuria (Table 1). Overall, 3 of 18 patients and 9 of 36 patients required emergency dialysis in group 1 and group 2, respectively $(p=0.73)$. 
Table 3. Kidney biopsy light microscopy characteristics of patients with IgG deposits (group 1)

\begin{tabular}{|c|c|c|c|c|c|c|c|c|c|c|c|c|}
\hline \multicolumn{13}{|c|}{ Kidney biopsy light microscopy } \\
\hline No. & gender & time & \multicolumn{6}{|c|}{ glomeruli } & ATN & $\begin{array}{l}\text { TAIF } \\
\text { grade }\end{array}$ & \multicolumn{2}{|c|}{ infiltrate } \\
\hline 1 & M & $02 / 02 / 11$ & 16 & 1 & 9 & no & S & 0 & no & III & yes & Mod \\
\hline 2 & M & $15 / 11 / 12$ & 6 & 4 & 0 & no & $\mathrm{F}$ & 0 & no & I & yes & Mod \\
\hline 5 & $\mathrm{M}$ & $25 / 01 / 13$ & 19 & 4 & 3 & yes & $S$ & 0 & no & $\mathrm{I}$ & yes & Mild \\
\hline 6 & $\mathrm{~F}$ & $15 / 05 / 09$ & 8 & 1 & 2 & yes & $\mathrm{C}$ & 0 & yes & II & yes & Mild \\
\hline 7 & $\mathrm{M}$ & $16 / 10 / 06$ & 29 & 5 & 4 & no & $\mathrm{C}$ & 1 & no & I & yes & Mod \\
\hline 8 & $\mathrm{~F}$ & $26 / 11 / 08$ & 20 & 0 & 14 & yes & $S$ & no & yes & II & yes & Sev \\
\hline 9 & $\mathrm{M}$ & $16 / 04 / 07$ & 15 & 2 & 2 & yes & S & no & yes & II & yes & Mild \\
\hline 14 & $\mathrm{~F}$ & $24 / 12 / 09$ & 7 & 0 & 4 & no & $\mathrm{S}$ & no & no & I & yes & Mod \\
\hline 15 & $\mathrm{~F}$ & $10 / 11 / 10$ & 16 & 3 & 3 & yes & $\mathrm{C}$ & no & yes & no & yes & Mild \\
\hline 16 & $\mathrm{M}$ & $31 / 03 / 09$ & 10 & 3 & 1 & yes & $\mathrm{C}$ & no & no & no & no & \\
\hline 17 & $\mathrm{M}$ & $31 / 08 / 11$ & 20 & 12 & 4 & no & $\mathrm{F}$ & no & no & I & yes & Mod \\
\hline 18 & $\mathrm{~F}$ & $24 / 09 / 09$ & 11 & 2 & 0 & yes & $\mathrm{C}$ & no & no & no & yes & Mod \\
\hline
\end{tabular}

Nx, normal; OG, obsolescent glomerulus; FN, fibrinoid necrosis; EUVAS; F, focal; S, sclerotic; M, mixt; C, crescentic; MHC, mesangial hypercellularity; ATN, acute tubular necrosis; TAIF, tubular atrophy and interstitial fibrosis; Infiltrate, cellular interstitial infiltrate; Mod, moderate; Sev, severe.

\section{Renal Pathology}

The two groups did not differ in mesangial hypercellularity $(p=0.418)$, fibrinoid necrosis $(p=0.777)$, or chronic vascular lesions $(p=0.569)$. Interstitial infiltrates, present in most patients of both groups, were often heterogeneous, with mainly lymphocytes, and occasional plasmacytes or histiocytes. Interstitial fibrosis and tubular atrophy lesions, which were moderate in $73 \%$ of patients, were more frequent in group 1 than in group 2 ( $p=0.024)$. However, acute tubular necrosis was more frequent in group 2 than in group $1(p=0.046)$.

Pathology characteristics of group 1 are presented in Table 3, and the deposits are detailed in Table 4. The staining score for the IgG deposits was 1 for 9 patients and $\geq 2$ in others. Among the 5 patients with an IgG staining score $\geq 3,4$ patients had kidney-limited vasculitis, and their creatinine level at diagnosis was not higher than that of other patients (median $259 \mu \mathrm{mol} / \mathrm{L}$ [133-412]). All had a positive C3 staining score $\geq 3$, and $3(60 \%)$ also had a $\mathrm{C} 1 \mathrm{q}$ staining score $\geq 3$. The location of IgG deposits, available for 16 patients, was parietal or in the glomerular basement membrane in 9 patients and mesangial in 7 pa- tients. The IgG staining was described as diffuse in 10 of $18(55 \%)$ patients of group 1. In addition, C3 component deposits were observed in all but 1 patient. IgM was also present in many patients, but IgA and C1q deposits were found unfrequently.

\section{Patient and Renal Outcomes}

Follow-up duration was similar in the two groups (median 30 months [7-81] for group 1 and 26 months [1299] for group $2 ; p=0.891$ ) and $>5$ years for 24 patients (6/18 in group 1 and $16 / 36$ in group 2$)$. Two patients in group 2 were lost to follow-up.

All deaths (group 1, $n=1$; group 2, $n=3$ ) were due to septic shock that occurred at $0.5,3,10$, and 48 months after treatment initiation. One infection occurred after surgery for a sigmoid adenocarcinoma. The two groups did not differ in patient survival $(p=0.828)$ (Fig. 2a).

Among the 12 patients who had initially undergone hemodialysis (group 1, $n=3$; group 2, $n=9$ ), 2 in each group recovered renal function during the early period. During follow-up, chronic hemodialysis was resumed in 2 patients. Finally, the two groups did not differ in renal 
Table 4. Characteristics of Ig and complement deposits in group 1

Kidney biopsy immunofluorescence

\begin{tabular}{|c|c|c|c|c|c|c|c|c|c|c|c|c|c|c|c|c|c|c|c|}
\hline \multirow[t]{2}{*}{ No. } & \multicolumn{4}{|c|}{ IgG } & \multicolumn{4}{|c|}{$\operatorname{IgA}$} & \multicolumn{4}{|c|}{ IgM } & \multicolumn{4}{|l|}{ C3 } & \multicolumn{3}{|c|}{$\mathrm{C} 1 \mathrm{q}$} \\
\hline & St. & Loc. & Kind & GD & St. & Loc. & Kind & GD & St. & Loc. & Kind & GD & St. & Loc. & Kind & GD & St. & Loc. & GD \\
\hline 1 & 1 & Glo & NA & Foc & 0 & & & & 1 & Foc & NA & Foc & 1 & Glo & NF & Foc & 0 & & \\
\hline 2 & 3 & Mes & NA & Diff & 1 & Mes & NA & Diff & 3 & Glo & NA & Foc & 4 & Mes & NA & Diff & 4 & Mes & Diff \\
\hline 3 & 3 & Mes & NA & Diff & 2 & Par & NA & Diff & 0 & & & & 3 & Mes & NA & Diff & 4 & Mes & Diff \\
\hline 4 & 1 & Mes & NA & Diff & 1 & Mes & NA & Diff & 2 & Glo & NA & Foc & 3 & Mes & NA & Diff & 1 & Glo & Foc \\
\hline 5 & 1 & Mes & NA & Diff & 0 & & & & 1 & Glo & NA & Foc & 3 & $\begin{array}{l}\text { Par/ } \\
\text { Mes }\end{array}$ & NA & Diff & 0 & & \\
\hline 6 & 2 & Mes & NA & Diff & 0 & & & & 0 & & & & 2 & Mes & NA & Diff & 0 & & \\
\hline 7 & 4 & Par & Gran & Diff & 0 & & & & 3 & Mes & NA & Diff & 4 & Par & Gran & Diff & 1 & Glo & Foc \\
\hline 8 & 1 & Par & NA & Foc & 0 & & & & 3 & Glo & NA & Foc & 1 & Glo & Gran & Foc & 1 & Glo & Foc \\
\hline 9 & 1 & Glo & NA & Foc & 0 & & & & 2 & Glo & $\mathrm{NF}$ & Foc & 3 & Mes & Gran & Diff & 1 & Glo & Foc \\
\hline 10 & 1 & Mes & NA & Foc & 0 & & & & 1 & Glo & NA & Foc & 1 & Glo & NA & Foc & 0 & & \\
\hline 11 & 2 & Mes & NA & Foc & 0 & & & & 3 & Mes & NA & Foc & 3 & Mes & NA & Foc & 2 & Mes & Foc \\
\hline 12 & 1 & Par & Gran & Diff & 0 & & & & 0 & & & & 4 & Par & Gran & Diff & 0 & & \\
\hline 13 & 3 & Par & Gran & Diff & 3 & Par & Gran & Diff & 1 & Par & Gran & Diff & 3 & Par & Gran & Diff & 0 & & \\
\hline 14 & 4 & GBM & Lin & Diff & 0 & & & & 0 & & & & 4 & GBM & Lin & Diff & 3 & GBM & I Diff \\
\hline 15 & 1 & Par & Gran & Foc & 3 & Par & Gran & Diff & 0 & & & & 3 & $\begin{array}{l}\text { Par/ } \\
\text { Mes }\end{array}$ & Gran & Foc & 0 & & \\
\hline 16 & 1 & Par & Gran & Foc & 3 & Par & Gran & Diff & 1 & Par & NA & Foc & 1 & Mes & Gran & Diff & 0 & & \\
\hline 17 & 2 & GBM & Lin & Foc & 0 & & & & 0 & & & & 1 & Glo & Lin & Foc & 0 & & \\
\hline 18 & 2 & GBM & Lin & Diff & 0 & & & & 0 & & & & 0 & & & & 0 & & \\
\hline
\end{tabular}

St., staining; Loc., localization inside the glomeruli; Glo, glomeruli without more precision; Mes, mesangial; Par, parietal; GBM, glomerular basement membrane; NA, not available; GD, glomerular diffusion; Gran, granular; Lin, linear; Foc, focal; Diff, diffuse.

Table 5. Relapses during follow-up

\begin{tabular}{llllll}
\hline & All & Group I & Group II & $p$ value \\
\hline Number of relapses (\%) & 0 & $42 / 54(78)$ & $15 / 18(84)$ & $27 / 36(75)$ & 0.73 \\
& 1 & $9(16)$ & $2(11)$ & $7(20)$ & 0.7 \\
& 2 & $2(4)$ & $1(5)$ & $1(2.5)$ & 1 \\
Kidney alone & 3 & $1(2)$ & $0(0)$ & $1(2.5)$ & 1 \\
& & $6 / 12(50)$ & $3 / 3(100)$ & $3 / 9(33)$ & 0.18 \\
\hline
\end{tabular}

survival (Fig. 2b), regardless of the use of plasma exchange ( $p=0.281$ ) or intensity of IgG staining. In addition, the two groups were similar in renal function (eGFR: $21.7 \pm 13.8$ vs. $28.3 \pm 24.1 \mathrm{~mL} / \mathrm{min} / 1.73 \mathrm{~m}^{2}$ for group $1 \mathrm{vs}$. group $2 ; p=0.515$ ) and proteinuria (Table 1). Creatinine level at last follow-up was similar for patients with a IgG staining score of 3 and lower scores.

Six of 12 relapses involved only the kidney (Table 5). The two groups did not differ in relapse frequency, but only renal limited relapses were observed in group 1 . The probability of relapse was similar between the two groups (Fig. 2c) $(p=0.422)$

\section{Discussion}

In this retrospective multicenter study, we report a cohort of ANCA-associated necrotizing CGN with glomerular IgG deposits seen on light microscopy. When compared to 36 patients with the classical pauci-immune histological form, this particular histological profile does not seem to identify a group of patients with specific initial characteristics or different outcomes.

The incidence of IgG deposits in AAV in previous studies based on light microscopy was highly variable $(2.2-28.5 \%)[9,10,15]$. Although we included patients 

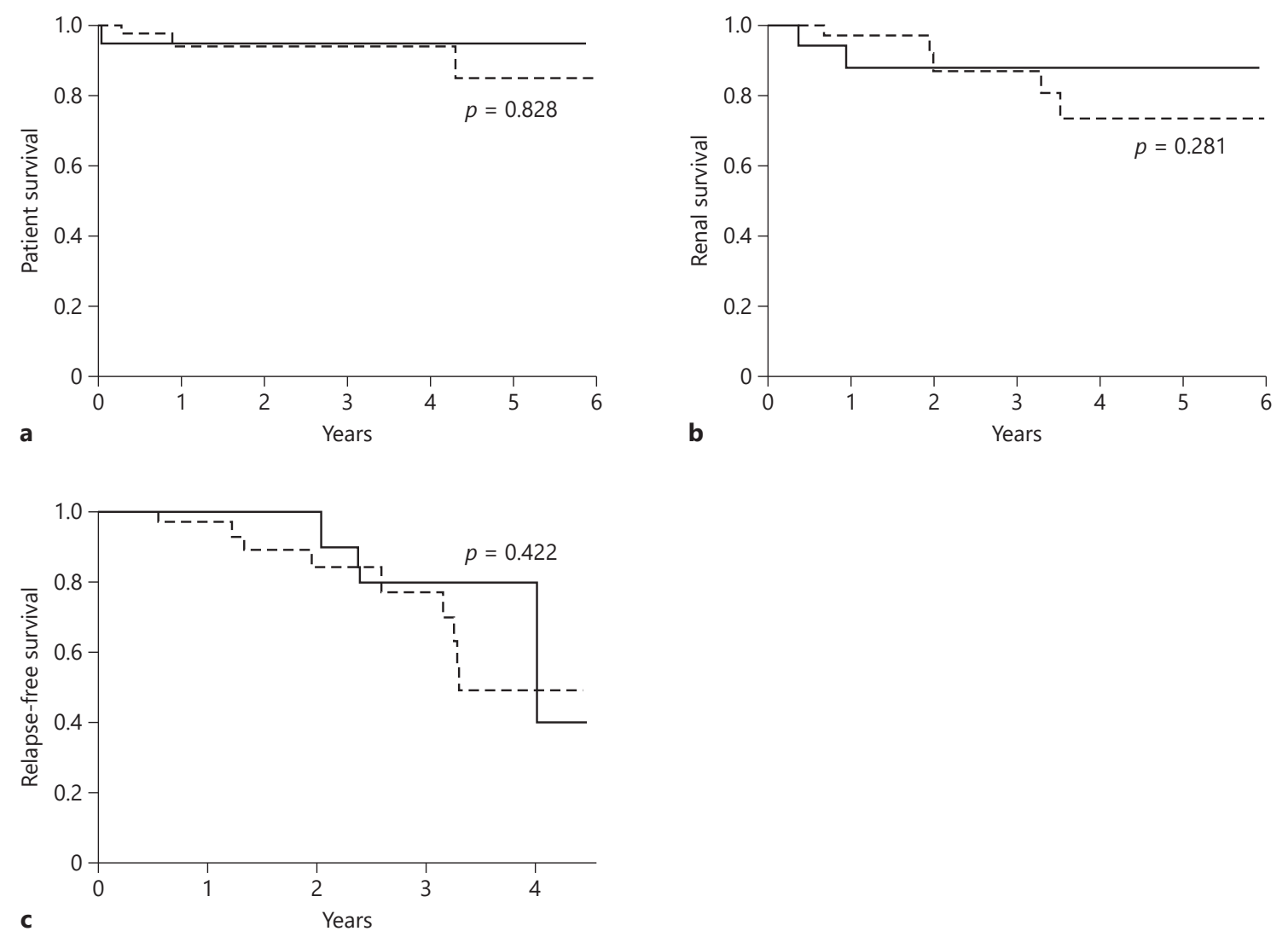

Fig. 2. The presence of glomerular IgG deposits had no negative impact on patient and renal survival. Estimated patient survival (a), renal survival (b), and relapse-free survival (c) in patients with primary ANCA-associated systemic vasculitis with (group 1, bold black line) and without (group 2, dashed line) renal IgG deposits.

with weak staining for IgG deposits, we report an overall incidence of $11 \%$. Thus, we confirmed that these deposits are not uncommon. In our cohort, half of the patients had only weakly stained IgG deposits. For many patients, IgG deposits were located along the glomerular basement membrane, but we also found a large proportion of deposits in the mesangium, as previously described $[9$, $10]$. Most of our patients with IgG deposits had MPOANCAs, in agreement with other studies $[8,9]$. Finally, the Ig deposits characteristics of our patients seemed similar to those reported in previous studies. Prior studies reported that patients with ANCA-associated CGN with IgG deposits had more severe renal disease, higher proteinuria [8-11], and more intense glomerular hypercellularity $[8,9]$ at the time of the diagnosis than those with pauci-immune ANCA-associated CGN. In our study, the level of proteinuria, renal function, and inten- sity of renal lesions of vasculitis did not differ between patients with ANCA-associated CGN with or without IgG deposits. Importantly, the rates of initial dialysis, remission, and relapses in our population were similar to those reported in large studies including patients with classical AAV [18-20]. In addition, we observed a similar spectrum of clinical presentation, in contrast to one study that suggested that systemic presentation would differ in patients with IgG deposits [11]. In agreement with our initial observations, we found that presence of glomerular IgG deposits did not affect renal outcome, as there was no difference in renal function or rate of ESRD between the groups after a median follow-up longer than 2 years. The evolution of AAV with IgG deposits has been described only once in 8 patients [10]. The authors reported that the creatinine level and the need for hemodialysis for ESRD tended to be higher within the first 2 
years in those patients compared to the control patients. The large proportion of patients with IgA deposits, that can evoke the presence of patients with ANCA-associated IgA, might explain these conflicting results. More generally, we think that it is important to take into account Ig classes and separately assess patients with IgG (possibly associated with other Ig), IgA, and IgM deposits, which could reflect different diseases with distinct pathological patterns. An original classification of patients with AAV and intrarenal Ig deposits has moreover been proposed with three groups [11]: group A1 with mesangial IgA and C3 deposits close to the IgA nephropathy phenotype, group A2 with mesangial IgG and C3 deposits, and group A3 with IgG and C3 deposits mainly in the capillary walls. Most of our patients would belong to group A2 or A3, and it would be interesting to assess whether renal outcome is affected by localization of $\operatorname{IgG}$ deposits, that could reflect different pathologies, such as membranous nephropathy (group A3) with a worse renal prognosis [21].

The absence of specificity regarding outcomes of patients with glomerular IgG deposits raises the question of specificity and pathogenicity of intrarenal IgG deposits. The specificity of IgG deposits in the kidney remains unknown. Our patients almost exclusively had MPOANCAs, mostly at very high titers, as compared to other studies [11]. MPO can be found directly on kidney biopsies and co-localizes with C3 and IgG [22] but only in glomerular lesions with low severity and activity. However, a few patients with PR3-ANCA were also included. A relationship between amounts of ANCAs, their titer, and the formation of deposits could also be assumed. For instance, patient No. 13 had important diffuse deposits at diagnosis. The disease evolution was marked by a persistent MPO-ANCA in blood and kidneys. Absence of any stigma of active disease on monitoring biopsies do not support a pathogenicity of antibodies in this patient. IgG deposits could also be more present during the first stages of the disease [22]. This corroborates with the shorter time to diagnosis of patients with IgG deposits ( 8 vs. 14 weeks, $p=0.131$ ). Nevertheless, higher incidence of acute tubular lesions and lower frequency of chronic tubular lesions for patients without IgG deposits did not support this temporal hypothesis.

Even though treatments were not statistically different between the two groups, we want to highlight that a slight larger proportion of patients with IgG deposits received plasma exchange and azathioprine, despite a selection of control patients based on date of diagnosis. We suspect that the presence of IgG deposits could directly impact the decision to use plasma exchanges, because clinicians fear a more severe disease [23] or possibly evoke another diagnosis such as anti-glomerular basement membrane disease. No explanation can be provided regarding the less frequent use of azathioprine in control patients. One clinical trial has suggested that plasma exchange would improve renal recovery in patients with ANCA-associated necrotizing CGN and creatinine $>500$ $\mu \mathrm{mol} / \mathrm{L}$ (less than $20 \%$ of patients in our study), but renal benefit was not confirmed after 4 years [23]. Renal survival was not improved in patients with plasma exchange in our cohort. Thus, it is unlikely that a more frequent use of plasma exchanges in patients with glomerular IgG deposits concealed a greater severity of renal disease in those patients. One clinical trial clearly showed that azathioprine was more effective than MMF to prevent relapse [24], possibly in patients with underexposure [25]. Nevertheless, the superiority of azathioprine over MMF remains unknown to prevent ESRD, and the risk of relapse did not differ in patients who were treated by azathioprine or MMF in our study (data not shown). Hence, we cannot specifically preclude that unbalanced use of azathioprine between groups has decreased the rate of relapse in patients with glomerular IgG deposits, but an absence of difference with regard to ESRD risk is unlikely related to it.

This study has several limitations. First, this was a retrospective case-control study with a relatively small number of patients with ANCA-associated CGN and IgG deposits, although it is currently the largest cohort. Because of our selecting strategy, baseline characteristics of groups were similar apart from the age of the patients. In addition, case-control selection within each center prevented bias due to the varied management of AAV in different units. We did not demonstrate a difference in outcomes between the two groups, in many instances because of the relatively small sample size and the low number of endpoints in survival analysis. This can be explained by the fact that it is a rare disease with a limited follow-up time, which corresponds to clinical practice.

Patients with IgG deposits more often received plasma exchange, which might be explained by the initial clinicians' "fear" of worse evolution associated with IgG deposits. Plasma exchange showed benefit in a clinical trial that included patients requiring dialysis or with serum creatinine $>500 \mu \mathrm{mol} / \mathrm{L}$ [26]. Only $17 \%$ of patients in group 1 and $19 \%$ in group 2 had a creatinine level $>500$ $\mu \mathrm{mol} / \mathrm{L}$, and renal survival was not improved in patients 
with plasma exchange in our cohort; therefore, our results are not likely affected by this difference between the two groups. Finally, pathological findings were based on reports analyzed by the same persons, and there was no centralized critical revision of slides. Ideally, electron microcopy should have been performed in every center, but no center was conducting routine electron microscopy to define the exact site of the complexes. The clinicians were confident with the diagnostic of ANCA-associated CGN, as lupus nephritis and anti-glomerular basement membrane disease were excluded.

In conclusion, IgG deposits are not uncommon in $\mathrm{AN}$ CA-associated CGN. Their presence does not change the clinical, biological, and histological pattern. Moreover, they did not affect renal and patient outcomes.

\section{Acknowledgements}

The authors would like to thank the "Societé des Nephrologues de l'Ouest" for this collaborative work.

\section{Statement of Ethics}

The authors have no ethical conflicts to disclose, this noninterventional study was conducted according to the French Ethics guidelines (before Jarde's Law 2016). All studies were performed in accordance with the Declaration of Helsinki.

\section{Disclosure Statement}

The authors have no conflicts of interest to declare.

\section{Funding Sources}

The authors have no funding to declare.

\section{Author Contributions}

All authors were involved in: conception or design, or analysis and interpretation of data, or both; drafting the article or revising it; providing intellectual content of critical importance to the work described; and final approval of the version to be published.

\section{References}

1 Jennette JC, Falk RJ, Bacon PA, Basu N, Cid MC, Ferrario F, et al. 2012 revised International Chapel Hill Consensus Conference Nomenclature of Vasculitides. Arthritis Rheum. 2013 Jan;65(1):1-11.

2 Savage CO, Winearls CG, Jones S, Marshall PD, Lockwood CM. Prospective study of radioimmunoassay for antibodies against neutrophil cytoplasm in diagnosis of systemic vasculitis. Lancet. 1987 Jun;1(8547):1389-93.

3 van der Woude FJ, Rasmussen N, Lobatto S, Wiik A, Permin H, van Es LA, et al. Autoantibodies against neutrophils and monocytes: tool for diagnosis and marker of disease activity in Wegener's granulomatosis. Lancet. 1985 Feb;1(8426):425-9.

4 Falk RJ, Jennette JC. Anti-neutrophil cytoplasmic autoantibodies with specificity for myeloperoxidase in patients with systemic vasculitis and idiopathic necrotizing and crescentic glomerulonephritis. N Engl J Med. 1988 Jun;318(25):1651-7.

5 Booth AD, Almond MK, Burns A, Ellis P, Gaskin G, Neild GH, et al.; Pan-Thames Renal Research Group. Outcome of ANCA-associated renal vasculitis: a 5-year retrospective study. Am J Kidney Dis. 2003 Apr;41(4):77684.

6 Slot MC, Tervaert JW, Franssen CF, Stegeman CA. Renal survival and prognostic factors in patients with PR3-ANCA associated vasculitis with renal involvement. Kidney Int. 2003 Feb;63(2):670-7.

7 Westman KW, Bygren PG, Olsson H, Ranstam J, Wieslander J. Relapse rate, renal survival, and cancer morbidity in patients with
Wegener's granulomatosis or microscopic polyangiitis with renal involvement. J Am Soc Nephrol. 1998 May;9(5):842-52.

8 Haas M, Eustace JA. Immune complex deposits in ANCA-associated crescentic glomerulonephritis: a study of 126 cases. Kidney Int. 2004 Jun;65(6):2145-52.

9 Yu F, Chen M, Wang SX, Zou WZ, Zhao MH, Wang HY. Clinical and pathological characteristics and outcomes of Chinese patients with primary anti-neutrophil cytoplasmic antibodies-associated systemic vasculitis with immune complex deposition in kidney. Nephrology (Carlton). 2007 Feb;12(1):7480.

10 Neumann I, Regele H, Kain R, Birck R, Meisl FT. Glomerular immune deposits are associated with increased proteinuria in patients with ANCA-associated crescentic nephritis. Nephrol Dial Transplant. 2003 Mar;18(3): 524-31.

11 Sumida K, Ubara Y, Nomura K, Hoshino J, Suwabe T, Hiramatsu R, et al. ANCA-associated crescentic glomerulonephritis with immune complex deposits. Clin Nephrol. 2012 Jun;77(6):454-60.

12 de Lind van Wijngaarden RA, Hauer HA, Wolterbeek R, Jayne DR, Gaskin G, Rasmussen $\mathrm{N}$, et al. Clinical and histologic determinants of renal outcome in ANCA-associated vasculitis: A prospective analysis of 100 patients with severe renal involvement. J Am Soc Nephrol. 2006 Aug; 17(8):2264-74.

13 Day CJ, Howie AJ, Nightingale P, Shabir S, Adu D, Savage CO, et al. Prediction of ESRD in pauci-immune necrotizing glomerulone- phritis: quantitative histomorphometric assessment and serum creatinine. Am J Kidney Dis. 2010 Feb;55(2):250-8.

14 Bollée G, Noël LH, Suarez F, Royal V, Gilardin L, de Serre NP, et al. Pauci-immune crescentic glomerulonephritis associated with ANCA of IgA class. Am J Kidney Dis. 2009 Jun;53(6): 1063-7.

15 Levey AS, Stevens LA, Schmid CH, Zhang YL, Castro AF 3rd, Feldman HI, et al.; CKD-EPI (Chronic Kidney Disease Epidemiology Collaboration). A new equation to estimate glomerular filtration rate. Ann Intern Med. 2009 May;150(9):604-12.

16 Berden AE, Ferrario F, Hagen EC, Jayne DR, Jennette JC, Joh K, et al. Histopathologic classification of ANCA-associated glomerulonephritis. J Am Soc Nephrol. 2010 Oct;21(10): 1628-36.

17 Mukhtyar C, Lee R, Brown D, Carruthers D, Dasgupta B, Dubey S, et al. Modification and validation of the Birmingham Vasculitis Activity Score (version 3). Ann Rheum Dis. 2009 Dec;68(12):1827-32.

18 Robson J, Doll H, Suppiah R, Flossmann O, Harper L, Höglund P, et al. Damage in the anca-associated vasculitides: long-term data from the European vasculitis study group (EUVAS) therapeutic trials. Ann Rheum Dis. 2015 Jan;74(1):177-84.

19 Jones RB, Tervaert JW, Hauser T, Luqmani $\mathrm{R}$, Morgan MD, Peh CA, et al.; European Vasculitis Study Group. Rituximab versus cyclophosphamide in ANCA-associated renal vasculitis. N Engl J Med. 2010 Jul;363(3): 211-20. 
20 Pierrot-Deseilligny Despujol C, Pouchot J, Pagnoux C, Coste J, Guillevin L. Predictors at diagnosis of a first Wegener's granulomatosis relapse after obtaining complete remission. Rheumatology (Oxford). 2010 Nov;49(11):2181-90.

21 Zou R, Liu G, Cui Z, Chen M, Zhao MH. Clinical and Immunologic Characteristics of $\mathrm{Pa}$ tients With ANCA-Associated Glomerulonephritis Combined With Membranous Nephropathy: A Retrospective Cohort Study in a Single Chinese Center. Medicine (Baltimore). 2015 Sep;94(37):e1472.

22 Kawashima S, Arimura Y, Sano K, Kudo A, Komagata Y, Kaname S, et al. Immunopathologic co-localization of MPO, IgG, and C3 in glomeruli in human MPO-ANCA-associated glomerulonephritis. Clin Nephrol. 2013 Apr; 79(4):292-301.

23 Walsh M, Casian A, Flossmann O, Westman K, Höglund P, Pusey C, et al.; European Vasculitis Study Group (EUVAS). Long-term follow-up of patients with severe ANCA-associated vasculitis comparing plasma exchange to intravenous methylprednisolone treatment is unclear. Kidney Int. 2013 Aug;84(2):397-402.

24 Hiemstra TF, Walsh M, Mahr A, Savage CO, de Groot K, Harper L, et al.; European Vasculitis Study Group (EUVAS). Mycophenolate mofetil vs azathioprine for remission maintenance in antineutrophil cytoplasmic anti- body-associated vasculitis: a randomized controlled trial. JAMA. 2010 Dec;304(21):2381-8.

25 Chaigne B, Gatault P, Darrouzain F, Barbet C, Degenne D, François M, et al. Mycophenolate mofetil in patients with anti-neutrophil cytoplasmic antibody-associated vasculitis: a prospective pharmacokinetics and clinical study. Clin Exp Immunol. 2014 May;176(2):172-9.

26 Jayne DR, Gaskin G, Rasmussen N, Abramowicz D, Ferrario F, Guillevin L, et al.; European Vasculitis Study Group. Randomized trial of plasma exchange or high-dosage methylprednisolone as adjunctive therapy for severe renal vasculitis. J Am Soc Nephrol. 2007 Jul;18(7):2180-8. 